\title{
946.
}

\section{NOTE ON THE THEORY OF ORTHOMORPHOSIS.}

[From the Quarterly Journal of Pure and Applied Mathematics, vol. xxvi. (1893), pp. 282-288.]

THE equation of any given curve whatever, $\Theta=0$, may be expressed in the form

$$
\phi(x+i y)+\phi(x-i y)=0 .
$$

Let $\chi$ be any odd function; then since

we have

$$
\phi(x-i y)=-\phi(x+i y)
$$

that is,

$$
\chi \phi(x-i y)=\chi\{-\phi(x+i y)\}=-\chi \phi(x+i y)
$$

$$
\chi \phi(x+i y)+\chi \phi(x-i y)=0
$$

Assuming that $\Theta$ is a real function, that is, a function with real coefficients, then also $\phi(x+i y)$ will be a function with real coefficients, or say a real function of $x+i y$; the function $\chi$ may be real or imaginary, but if imaginary, then the $i$ of the coefficients does not change its sign in the passage from $\chi \phi(x+i y)$ to $\chi \phi(x-i y)$.

In proof of the assumed theorem, imagine the equation $\Theta=0$ expressed as an equation between $x+i y$ and $x-i y$, or, supposing it solved in regard to $x-i y$, take the form of it to be $x-i y=f(x+i y)$ : let $u_{n}$ be a function of $n$ satisfying the equation of differences $u_{n+1}=f u_{n}$; and let $\phi(x+i y)$ be determined as a function of $x+i y$ by the elimination of $n$ from the equations

we thence have

$$
x+i y=u_{n}, \quad \phi(x+i y)=\cos n \pi ;
$$

and consequently

$$
x-i y=f u_{n},=u_{n+1},
$$

$$
\phi(x-i y)=\cos (n+1) \pi,
$$


that is,

$$
\phi(x+i y)+\phi(x-i y)=0,
$$

viz. this equation is a transformation of the equation $\Theta=0$, and thus it appears that the equation $\Theta=0$ can always be thrown into the last-mentioned form.

As an example, take the equation $y=a x+b$ : which, putting for a moment $\xi=x+i y, \eta=x-i y$, is

$$
\frac{1}{2 i}(\xi-\eta)=\frac{1}{2} a(\xi+\eta)+b
$$

that is,

$$
\eta=\frac{i+a}{i-a} \xi+\frac{2 b}{i-a}
$$

we have therefore

$$
u_{n+1}=\frac{i+a}{i-a} u_{n}+\frac{2 b}{i-a},
$$

a solution of which is

$$
u_{n}=\left(\frac{i+a}{i-a}\right)^{n}-\frac{b}{a}
$$

putting this $=\xi$, we have

$$
n=\frac{1}{\log \frac{i+a}{i-a}} \log \left(\xi+\frac{b}{a}\right)
$$

and thence

$$
\phi \xi=\cos \frac{\pi}{\log \frac{i+a}{i-a}} \log \left(\xi+\frac{b}{a}\right),
$$

where observe that, writing $a+i=R e^{i a}$ and therefore $a-i=R e^{-i a}$, we have

$$
\cos \alpha=\frac{a}{\sqrt{ }\left(a^{2}+1\right)}, \quad \sin \alpha=\frac{1}{\sqrt{ }\left(a^{2}+1\right)},
$$

or say $\cot \alpha=a$, and then

$$
\frac{i+a}{i-a}=e^{2 \alpha i+i \pi}, \text { or } \log \frac{i+a}{i-a}=i(2 \alpha+\pi)
$$

whence

$$
\phi \xi=\cos \frac{\pi}{i(2 \alpha+\pi)} \log \left(\xi+\frac{b}{a}\right),=\cosh \frac{\pi}{2 \alpha+\pi} \log \left(\xi+\frac{b}{a}\right),
$$

a real function of $\xi$.

In verification of the equation $\phi \xi+\phi \eta=0$, we have

$$
\phi \eta=\cos \frac{\pi}{\log \frac{i+a}{i-a}} \log \left(\eta+\frac{b}{a}\right),
$$


where

and thence

$$
\begin{aligned}
\log \left(\eta+\frac{b}{a}\right) & =\log \left(\frac{i+a}{i-a} \xi+\frac{2 b}{i-a}+\frac{b}{a}\right)=\log \frac{i+a}{i-a}\left(\xi+\frac{b}{a}\right) \\
& =\log \frac{i+a}{i-a}+\log \left(\xi+\frac{b}{a}\right)
\end{aligned}
$$

$$
\begin{aligned}
\phi \eta & =\cos \frac{\pi}{\log \frac{i+a}{i-a}}\left\{\log \frac{i+a}{i-a}+\log \left(\xi+\frac{b}{a}\right)\right\} \\
& =\cos \left\{\pi+\frac{\pi}{\log \frac{i+a}{i-a}} \log \left(\xi+\frac{b}{a}\right)\right\},=-\cos \frac{\pi}{\log \frac{i+a}{i-a}} \log \left(\xi+\frac{b}{a}\right),
\end{aligned}
$$

that is, $\phi \eta=-\phi \xi$, or $\phi \xi+\phi \eta=0$, the equation in question.

I remark, in passing, that the same equation $y=a x+b$ might have been put in the form $\phi x+\phi y=0$, viz. assuming

then

$$
\phi x=\cos \frac{\pi}{\log a} \log \left(x-\frac{b}{1-a}\right),
$$

that is, $\phi x+\phi y=0$.

$$
\begin{aligned}
\phi y & =\cos \frac{\pi}{\log a} \log \left(a x+b-\frac{b}{1-a}\right)=\cos \frac{\pi}{\log a} \log a\left(x-\frac{b}{1-a}\right) \\
& =\cos \frac{\pi}{\log a}\left\{\log a+\log \left(x-\frac{b}{1-a}\right)\right\} \\
& =\cos \left\{\pi+\frac{\pi}{\log a} \log \left(x-\frac{b}{1-a}\right)\right\} \\
& =-\cos \frac{\pi}{\log a} \log \left(x-\frac{b}{1-a}\right)=-\phi x,
\end{aligned}
$$

If $b=0$, then

$$
y=a x \text { and } \phi x=\cos \frac{\pi \log x}{\log a} ;
$$

in fact, repeating the proof for this particular case,

that is,

$$
\phi y=\cos \pi \frac{\log a x}{\log a}=\cos \pi\left(1+\frac{\log x}{\log a}\right)=-\cos \frac{\pi \log x}{\log a}, \quad=-\phi x
$$

$$
\phi x+\phi y=0 .
$$

Considering then $(x, y)$ as the coordinates of a point on the curve $\Theta=0$, we have, as above,

$$
\chi \phi(x+i y)+\chi \phi(x-i y)=0,
$$

where $\phi$ is a real function determined as above, and $\chi$ is any real or imaginary odd function. This being so, assume

$$
x_{1}+i y_{1}=e^{x(x+i y)} \text {, }
$$


then also

and consequently

$$
x_{1}-i y_{1}=e^{X \phi(x-i y)} \text {, }
$$$$
x_{1}^{2}+y_{1}^{2}=e^{\chi \phi(x+i y)+\chi \phi(x-i y)}=1 \text {, }
$$

that is, we have the circumference of the circle $x_{1}{ }^{2}+y_{1}{ }^{2}-1=0$ corresponding to the given curve $\Theta=0$.

Suppose that the curve $\Theta=0$ is a closed curve: and then writing

and therefore

$$
\xi+i \eta=\chi \phi(x+i y)
$$

we thence have

$$
\xi-i \eta=\chi \phi(x-i y)
$$

a real function of $(x, y)$.

$$
2 \xi=\chi \phi(x+i y)+\chi \phi(x-i y)
$$

(1) Assume that it is possible to find $\chi$, such that $\xi$ as defined by this last equation shall be throughout the area of the curve $\Theta=0$ finite and continuous, except only that in the neighbourhood of a given point, taken to be the point $x=0, y=0$, it is $=\log \sqrt{ }\left(x^{2}+y^{2}\right)$.

(2) At the boundary of the area $\Theta=0, \xi$ is $=0$.

(3) Throughout the area, $\xi$ satisfies the partial differential equation

$$
\frac{d^{2} \xi}{d x^{2}}+\frac{d^{2} \xi}{d y^{2}}=0
$$

These conditions being satisfied, the equation

that is,

$$
x_{1}+i y_{1}=e^{\xi+i \eta} \text {, }
$$

$$
x_{1}+i y_{1}=e^{\chi \phi(x+i y)} \text {, }
$$

gives an orthomorphosis of the area $\Theta=0$ into the circle $x_{1}^{2}+y_{1}{ }^{2}-1=0$, the point $x=0, y=0$ corresponding to the centre of the circle; (2) and (3) are satisiied as above: it remains only to satisfy (1), viz. the function $\chi$ is determined not by any equationbut only by this condition as to finiteness and continuity; and if it be thus determined, then the foregoing equation $x_{1}+i y_{1}=e^{x \phi(x+i y)}$ gives the required orthomorphosis.

For instance, let the curve $\Theta=0$ be the parabola $y^{2}=4(1-x)$, which may be regarded as a closed curve bounding the infinite parabolic area. We have $2 x=\xi+\eta$, $2 i y=\xi-\eta$, whence the equation is

that is,

$$
-\frac{1}{4}(\xi-\eta)^{2}=4-2 \xi-2 \eta,
$$

$$
\xi^{2}-2 \xi \eta+\eta^{2}-8 \xi-8 \eta+16=0,
$$

whence $\sqrt{ } \xi+\sqrt{ } \eta-2=0$, or writing this in the form

$$
(\sqrt{ } \xi-1)+(\sqrt{ } \eta-1)=0
$$


we have $\phi \xi=\sqrt{ } \xi-1$, and assuming that $\chi$ can be found so that the condition as to finiteness and continuity is satisfied, then the orthomorphosis is given by

$$
x_{1}+i y_{1}=\exp \chi(\sqrt{ } \xi-1),=\exp \chi\{\sqrt{ }(x+i y)-1\} .
$$

Assuming

$$
\frac{1}{2} \chi \omega=-\frac{1}{2} i \pi \omega+\log \frac{1-i \exp \left(\frac{1}{2} i \pi \omega\right)}{1-i \exp \left(-\frac{1}{2} i \pi \omega\right)}
$$

which is obviously an odd function, we have

which is

$$
\begin{aligned}
\exp \frac{1}{2} \chi \omega & =\frac{1}{\exp \frac{1}{2} i \pi \omega} \frac{1-i \exp \left(\frac{1}{2} i \pi \omega\right)}{1-i \exp \left(-\frac{1}{2} i \pi \omega\right)}, \\
& =\frac{1-\exp \frac{1}{2} i \pi \omega}{\exp \frac{1}{2} i \pi \omega-1},=\frac{i\left(1-i \exp \frac{1}{2} i \pi \omega\right)}{1+i \exp \frac{1}{2} i \pi \omega}, \\
& =\tan \frac{1}{4} \pi(\omega+1),
\end{aligned}
$$

and hence, for $\omega$ writing $\sqrt{ }(x+i y)-1$, we have

$$
x_{1}+i y_{1}=\exp \chi\{\sqrt{ }(x+i y)-1\}, \quad=\tan ^{2} \frac{1}{4} \pi \sqrt{ }(x+i y)
$$

This satisfies the required conditions as to finiteness and continuity; and in particular, we have

$$
\xi+i \eta=\log \tan ^{2} \frac{1}{4} \pi \sqrt{ }(x+i y)
$$

so that, $x$ and $y$ being small,

that is,

$$
\xi+i \eta=\log \frac{\pi^{2}}{16}(x+i y), \quad \xi-i \eta=\log \frac{\pi^{2}}{16}(x-i y)
$$

$$
\xi=\log \frac{\pi^{2}}{16} \sqrt{ }\left(x^{2}+y^{2}\right)
$$

Hence we have the known result: the orthomorphosis of the parabola $y^{2}=4(1-x)$ into the circle $x_{1}^{2}+y_{1}^{2}-1=0$ is given by the equation $x_{1}+i y_{1}=\tan ^{2} \frac{1}{4} \pi \sqrt{ }(x+i y)$.

Consider the ellipse, where $a^{2}-b^{2}=1$, or say

$$
\frac{x^{2}}{\frac{1}{4}\left(M+\frac{1}{M}\right)^{2}}+\frac{y^{2}}{\frac{1}{4}\left(M-\frac{1}{M}\right)^{2}}=1 \text {. }
$$

I show, by a less direct process, how to express this equation in the required form $\phi \xi+\phi \eta=0$. In fact, writing

$$
\xi=x+i y, \quad \eta=x-i y,
$$

the equation of the ellipse is the rationalised form of

$$
i \eta+\sqrt{ }\left(1-\eta^{2}\right)=M^{2}\left\{i \xi+\sqrt{ }\left(1-\xi^{2}\right)\right\} .
$$


To show that this is so, call for a moment the right-hand side $\Omega$, the equation is

$$
\sqrt{ }\left(1-\eta^{2}\right)=\Omega-i \eta
$$

hence

or

$$
\begin{aligned}
1-\eta^{2} & =\Omega^{2}-2 \Omega i \eta-\eta^{2} \\
2 \Omega i \eta & =\Omega^{2}-1,
\end{aligned}
$$

therefore

$$
\begin{aligned}
2 i \eta^{*}=\Omega-\frac{1}{\Omega} & =M^{2}\left\{i \xi+\sqrt{ }\left(1-\xi^{2}\right)\right\}+\frac{1}{M^{2}}\left\{i \xi-\sqrt{ }\left(1-\xi^{2}\right)\right\} \\
& =\left(M^{2}+\frac{1}{M^{2}}\right) i \xi+\left(M^{2}-\frac{1}{M^{2}}\right) \sqrt{ }\left(1-\xi^{2}\right),
\end{aligned}
$$

$$
\begin{gathered}
2 i \eta-\left(M^{2}+\frac{1}{M^{2}}\right) i \xi=\left(M^{2}-\frac{1}{M^{2}}\right) \sqrt{ }\left(1-\xi^{2}\right) \\
-4 \eta^{2}+4\left(M^{2}+\frac{1}{M^{2}}\right) \xi \eta+\left(M^{4}+2+\frac{1}{M^{4}}\right)(-\xi)^{2}=\left(M^{4}-2+\frac{1}{M^{4}}\right)-\left(M^{4}-2+\frac{1}{M^{4}}\right) \xi^{2}
\end{gathered}
$$

that is,

$$
-4 \eta^{2}-4 \xi^{2}+4\left(M^{2}+\frac{1}{M^{2}}\right) \xi \eta=\left(M^{2}-\frac{1}{M^{2}}\right)^{2}
$$

or say

$$
-\xi^{2}-\eta^{2}+\left(M^{2}+\frac{1}{M^{2}}\right) \xi \eta-\frac{1}{4}\left(M^{2}-\frac{1}{M^{2}}\right)^{2}=0:
$$

viz. substituting for $\xi, \eta$ their values, this is

$$
-2\left(x^{2}-y^{2}\right)+\left(M^{2}+\frac{1}{M^{2}}\right)\left(x^{2}+y^{2}\right)-\frac{1}{4}\left(M^{2}-\frac{1}{M^{2}}\right)^{2}=0,
$$

that is,

$$
\left(M-\frac{1}{M}\right)^{2} x^{2}+\left(M+\frac{1}{M}\right)^{2} y^{2}-\frac{1}{4}\left(M^{2}-\frac{1}{M^{2}}\right)^{2}=0,
$$

or finally, it is

$$
\frac{x^{2}}{\frac{1}{4}\left(M+\frac{1}{M}\right)^{2}}+\frac{y^{2}}{\frac{1}{4}\left(M-\frac{1}{M}\right)^{2}}-1=0
$$

as it should be.

Starting then from the relation

$$
i \eta+\sqrt{ }\left(1-\eta^{2}\right)=M^{2}\left\{i \xi+\sqrt{ }\left(1-\xi^{2}\right)\right\},
$$

and writing

$$
\phi \xi=\cos \frac{\pi}{2 \log M} \log \left\{i \xi+\sqrt{ }\left(1-\xi^{2}\right)\right\},
$$


we have

$$
\begin{aligned}
\phi \eta & =\cos \frac{\pi}{2 \log M} \log M^{2}\left\{i \xi+\sqrt{ }\left(1-\xi^{2}\right)\right\} \\
& =\cos \frac{\pi}{2 \log M}\left[\log M^{2}+\log \left\{i \xi+\sqrt{ }\left(1-\xi^{2}\right)\right\}\right] \\
& =\cos \left[\pi+\frac{\pi}{2 \log M} \log \left\{i \xi-\sqrt{ }\left(1-\xi^{2}\right)\right\}\right] \\
& =-\cos \frac{\pi}{2 \log M} \log \left\{i \xi+\sqrt{ }\left(1-\xi^{2}\right)\right\},=-\phi \xi
\end{aligned}
$$

that is, we have

$$
\phi \xi+\phi \eta=0
$$

as the required transformation of the equation of the ellipse

$$
\frac{x^{2}}{\frac{1}{4}\left(M+\frac{1}{M}\right)^{2}}+\frac{y^{2}}{\frac{1}{4}\left(M-\frac{1}{M}\right)^{2}}=1 .
$$

We hence derive the known formula for the orthomorphosis of the ellipse into the circle $x_{1}^{2}+y_{1}^{2}-1=0$. 